\title{
Gamma-ray observations at the coastal area of Japan Sea in winter seasons
}

\author{
H. Tsuchiya ${ }^{*}$, , T. Enoto, ${ }^{b, c}$ Y. Wada, ${ }^{c, d}$, Y. Furuta, ${ }^{e}$ K. Nakazawa, ${ }^{f}$ T. Yuasa, ${ }^{g}$ D. \\ Umemoto, ${ }^{h}$ K. Makishima ${ }^{i}$, and the GROWTH collaboration \\ ${ }^{a}$ Nuclear Science and Engineering Center, Japan Atomic Energy Agency \\ ${ }^{b}$ The Hakubi Center for Advanced Research and Department of Astronomy, Kyoto University \\ ${ }^{c}$ High Energy Astrophysics Laboratory, RIKEN Nishina Center \\ ${ }^{d}$ Department of Physics, Graduate School of Science, The University of Tokyo \\ ${ }^{e}$ Collaborative Laboratories for Advanced Decommissioning Science, Japan Atomic Energy \\ Agency \\ ${ }^{f}$ Kobayashi-Maskawa Institute for the Origin of Particles and the Universe, Nagoya university \\ ${ }^{g}$ Block 4B, Boon Tiong Road,Singapore 165004, Singapore \\ ${ }^{h}$ Advanced Institute for Computational Science, RIKEN \\ ${ }^{i}$ Kavli Institute for the Physics and Mathematics of the Universe, The University of Tokyo \\ E-mail: tsuchiya.harufumi@jaea.go.jp
}

Since 2006, The Gamma Ray Observation of Winter Thunderclouds (GROWTH) collaboration has operated radiation measurement networks at the coastal area of Japan sea. The area is famous for its frequent occurrence of winter thunderstorms. We aims at elucidating how particles in lightning and thunderclouds are accelerated to relativistic energies. More than 10-years observations reveal that there are two types of radiation bursts associated with winter thunderstorms. One is a "long burst" or a "gamma-ray glow" lasting for a few tens of seconds to a few minutes. The other is a "short burst" in association with lightning. In order to expand the observational network, we have developed a small type of a radiation detector. In this paper, we focus on recent several findings obtained by the new detectors. One is a combination of a short burst and a long one, showing simultaneous detection of prompt gamma rays extending to $\sim 10 \mathrm{MeV}$ and the 511-keV annihilation ones. These gamma-ray signals demonstrate the occurrence of photonuclear reactions in lightning. Another is an event that a gamma-ray glow suddenly ceased just prior to lightning, suggesting a relation between lightning and the two types of radiation bursts. On the basis of these results, we discuss the production mechanism of gamma rays related to thunderstorms and the lightning initiation problem.

36th International Cosmic Ray Conference -ICRC2019-

July 24th - August 1st, 2019

Madison, WI, U.S.A.

${ }^{*}$ Speaker. 


\section{Introduction}

In 1925, C. T. R. Wilson proposed that electrons are accelerated to high energy in strong electric fields of thunderclouds and produce $\mathrm{x}$ rays via bremsstrahlung [1]. Since then, many observations were made to measure such $\mathrm{x}$-ray signals, revealing that lightning as well as thunderclouds are powerful particle accelerators [2]. Interestingly, beyond the Wilson's prediction, high-energy radiations with a few tens of $\mathrm{MeV}$ have been detected from lightning and thunderclouds, suggesting that electrons can be accelerated beyond a few tens of $\mathrm{MeV}$ therein. In order to explain the production mechanism of those high-energy radiation, theoretical works $[3,4]$ have postulated the presence of relativistic runaway electrons. The runaway electrons have been thought to be responsible for various high-energy phenomena during thunderstorms.

With its duration, observed radiation during thunderstorms can be categorized into two types. One is an intense lightning-related emission in short pulses ranging from microsecond to millisecond order. The other is a gamma-ray glow, being a relatively faint emission roughly lasting for minute order. The former has been frequently observed by satellites, called terrestrial gamma-ray flashes (TGFs) [5, 6]. On the ground, high-energy emissions are also observed in coincidence with natural and rocket-triggered lightning. Because lightning-related emission produce a huge photon flux in short duration of $\sim 1 \mathrm{~ms}$ or less to often saturate detectors, its complete picture has been hardly obtained so far.

Gamma-ray glows (also referred to as long bursts or terrestrial gamma-ray enhancements) have been observed by airplanes [7, 8], high-mountain detectors $[9,10,11,12,13]$ and sea-level ones $[14,15,16]$. Although those glows do not generally coincide with lightning, several glow observations confirmed that a glow precedes lightning [7, 17, 18, 8, 19], indicating a close relation with lightning initiation. Those observations provide one possibility that a glow always occurs before lightning. Whether this possibility is true or not remains an open question.

In this proceeding, we show recent observational results obtained by Gamma-Ray Observation of Winter THunderclouds (GROWTH) collaboration at the coastal area of Japan sea, including Enoto et al.(2017) [20] and Wada et al.(2019) [21]. With the results, we will discuss high-energy atmospheric phenomena including photonuclear reactions in lightning and termination of a gammaray glow.

\section{Experiments at the coastal area of Japan Sea}

For the purpose of understanding high-energy atmospheric phenomena related to thunderstorms, the GROWTH collaboration has operated radiation measurement system at the coastal area of Japan sea in Prefectures of Niigata and Ishikawa for $>10$ years. Details on the GROWTH experiment are found in $[19,20]$ and hence a brief explanation are given here.

In 2006, we started high-energy radiation measurement at the Kashiwazaki-Kariwa nuclear power plant facing on the coastal area of Japan sea. Winter thunderstorms, which are rare in the world, frequently occur around the coastal area. In addition, base altitude of winter thunderclouds, $1 \mathrm{~km}$ or less is lower than summer ones, which is a suitable condition to observe radiation emitted from lightning and thunderclouds. Radiation systems, equipped with gamma-ray detectors and 

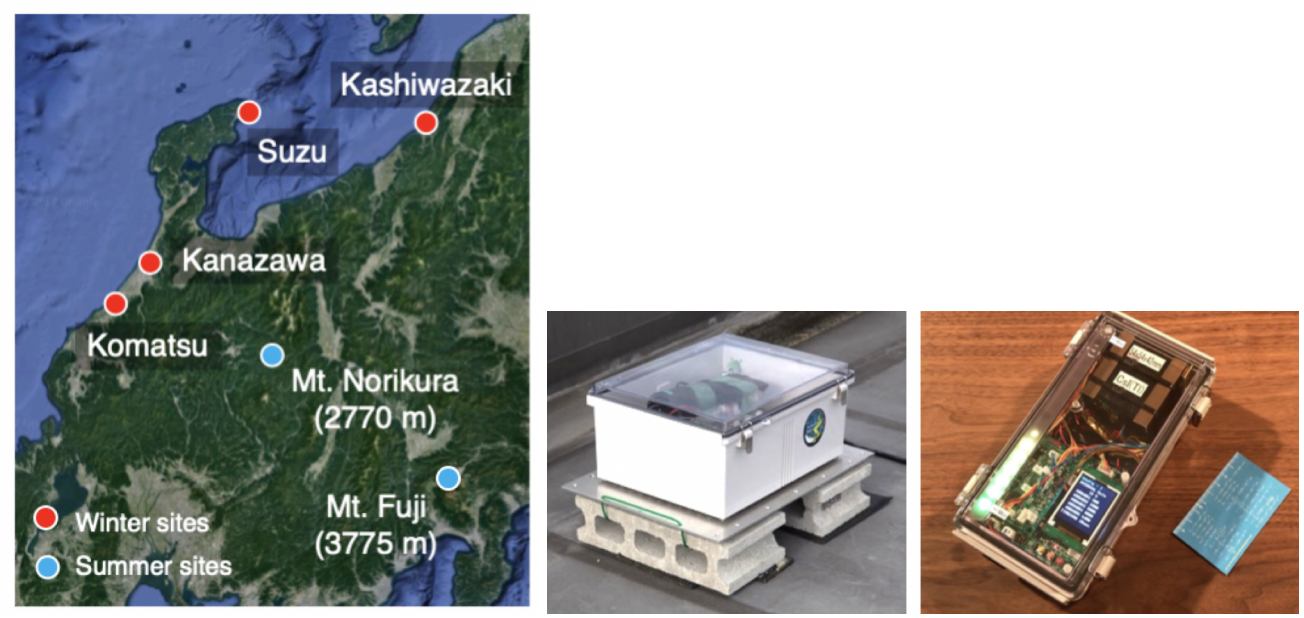

Figure 1: (left) Observational network in 2018. (right) Radiation measurement system installed at individual sites.

environmental monitors, were installed in the plant and successfully detected radiation bursts [15, 16]. In the plant we were able to obtain 1-2 radiation events yearly.

To increase the number of radiation events observed and better elucidate the mechanisms of radiation bursts, we have expanded our observation network. Figure 1 shows the observation sites around the coastal area of Japan sea in the 2018 winter season. As a reference, summer observation sites are also shown. the GROWTH collaboration currently uses a small-type of radiation measurement system (Fig.1). To detect $\mathrm{x}$ rays and gamma rays, the small system has an inorganic scintillator such as BGO or CsI ones. Observations, described later, employed $25 \mathrm{~cm} \times 8 \mathrm{~cm} \times$ $2.5 \mathrm{~cm}$ BGO scintillators. These small type radiation detection systems have been arranged at sites indicated by red points of Fig. 1 and the total number of the detection system installed was 23 in the 2018 winter season.

\section{Results}

\subsection{Photonulcear reactions in lightning}

C .T. R. Willson already predicted in 1920 's that nuclear reactions may take place in lightning [1]. Libby \& Lukens [22] also showed the possibility that lightning could produce neutrons via $\mathrm{D}+\mathrm{D} \rightarrow \mathrm{n}+{ }^{3} \mathrm{He}$ to contribute partly to radiocarbon in tree rings. Then, the first positive neutron detection was reported in 1980's by Shah et al. [23]. However, Babich et al., [24] indicated that it would be quite difficult for the DD reactions to occur in a normal lightning environment. Instead of the DD reactions, they proposed that a feasible channel to generate neutrons is a photonuclear reaction with its threshold of $10.6 \mathrm{MeV}$ for nitrogen in the atmosphere, ${ }^{14} \mathrm{~N}+\gamma \rightarrow{ }^{13} \mathrm{~N}+\mathrm{n}$.

On February 6, 2017, we observed an intense radiation burst associated with lightning, using 4 detectors installed at Kashiwazaki-Kariwa nulcear power plant. Though initial radiations coming to the detectors paralyzed them, a decaying phase was found in the time profile $\sim 20 \mathrm{~ms}$ after the initial radiations. Figure 2 shows a count history and energy spectra observed by one detector (detector-A) in this decaying phase. The decay component of the count history can be expressed by 
an exponential form with a decay constant of $56 \pm 3 \mathrm{~ms}$. Unlike an energy spectrum of gamma-ray glows observed so far $[15,16]$, the observed spectrum showed a cutoff of $\sim 10 \mathrm{MeV}$. In addition, annihilation signals of $0.51 \mathrm{MeV}$ were measured for $\sim 1$ minute.

Assuming photonulcear reaction occurred in lightning, we can explain the observed features. High-energy electrons accelerated to a few tens of $\mathrm{MeV}$ in lightning produce bremsstrahlung emissions with energy exceeding $10.6 \mathrm{MeV}$. The bremsstrahlung emissions generate fast neutrons via photonuclear reactions of ${ }^{14} \mathrm{~N}+\gamma \rightarrow{ }^{13} \mathrm{~N}+\mathrm{n}$. The produced fast neutrons are repeatedly scattered in the atmosphere, moderated toward lower-energy enough to be captured by atmospheric nuclei. Then, prompt gamma rays are emitted via neutron capture reactions such as ${ }^{14} \mathrm{~N}+\mathrm{n} \rightarrow{ }^{15} \mathrm{~N}^{*}+\gamma$. The neutron capture gamma rays derived from ${ }^{14} \mathrm{~N}$ have the maximum energy of $10.8 \mathrm{MeV}$. According to a numerical calculation, a moderation time that a $10-\mathrm{MeV}$ neutron reduces its energy to $1 \mathrm{eV}$ turns to be $56 \mathrm{~ms}$, consistent with the observed time scale of the decaying phase. Furthermore, annihilation lines of $0.51 \mathrm{MeV}$ are also attributable to photonulcear reactions. Residual ${ }^{13} \mathrm{~N}$ has a half-life of $\sim 10 \mathrm{~min}$, emitting a positron via beta-plus decay of ${ }^{13} \mathrm{~N} \rightarrow{ }^{13} \mathrm{C}+e^{+}+v_{e}$. The positrons can produce $0.51 \mathrm{MeV}$ annihilation lines via $e^{+} e^{-}$in the atmosphere. The reason why the lines gave our detectors signals for $\sim 1$ minutes is that thunderclouds containing ${ }^{13} \mathrm{~N}$ passed over our detectors with emitting the lines.
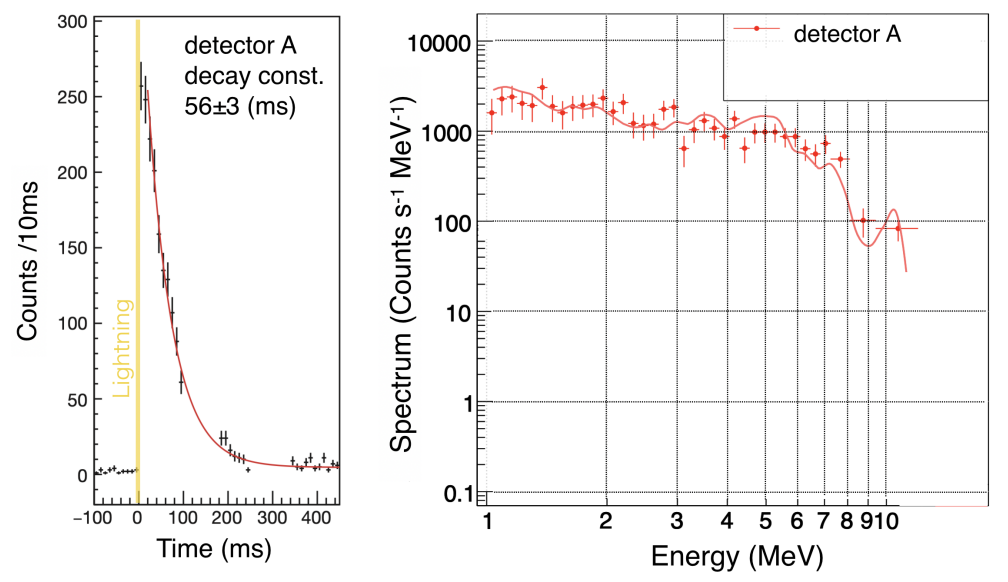

Figure 2: (left) $>0.35 \mathrm{MeV}$ count history of detector-A. A red line shows the best fitting function of an exponential decay. (right) energy spectrum obtained in the decaying phase (40 ms-200 ms). A red line corresponds to results of the GEANT4 simulation.

From this observation, we expect that photonuclear reactions in lightning result in production of ${ }^{14} \mathrm{C}$ [20]. This is because total cross section of ${ }^{14} \mathrm{~N}(n, p){ }^{14} \mathrm{C}$ is $\sim 25$ times larger than that of neutron capture reaction in thermal to epithermal energy region. Therefore, lightning in addition to cosmic rays would be the second natural source of ${ }^{14} \mathrm{C}$.

\subsection{Termination of a gamma-ray glow}

Figure 3 shows 1-s count histories of radiation detectors installed in Kanazawa city, obtained on 2018 January 9 with two detectors shown in Figure 4. Detector-A first measured the glow and then detector-B also did $\sim 30 \mathrm{sec}$ after the commencement of the enhancement of detector-A. This delay corresponds to movement of thunderclouds in direction denoted in Fig 4. In addition, the 

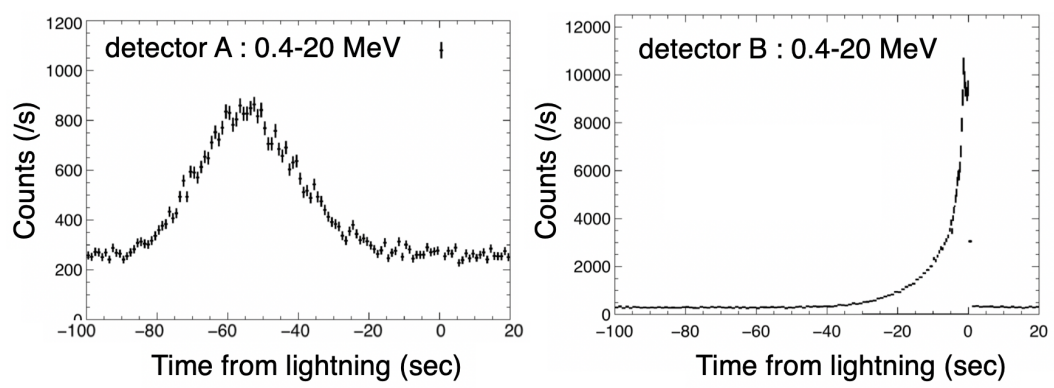

Figure 3: One-sec count histories obtained by detectors A (left) and B(right).

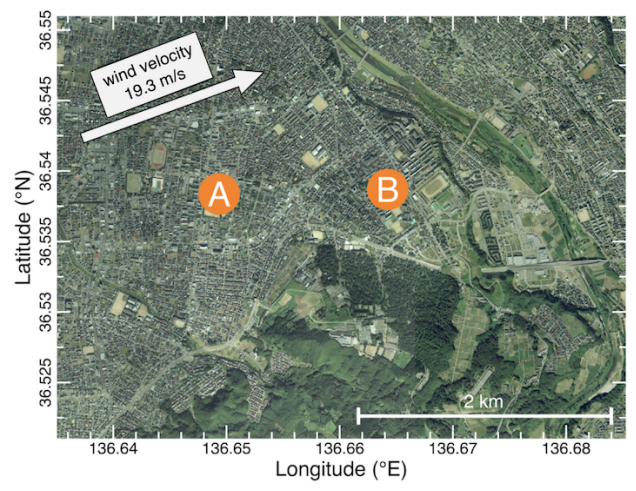

Figure 4: Detector positions in Kanazawa city. Image was provided by the Geospatial Information Authority of Japan.

peak of the count increase of detector-B is $>10$ times higher than that of detector-A, suggesting a central position of the gamma-ray glow would be closer to detector-B rather than detector-A. As shown in Fig. 3, counts of detector-B quickly dropped to the background level after lightning. Such a termination of a gamma-ray glow has been observed during past observations including the GROWTH events [7, 17, 18]. Unlike past termination events, the two detectors-A and -B registered a short burst lasting for $\sim 0.2 \mathrm{~s}$ just after lightning. At the very beginning of the short burst, both detectors registered signs of an extremely large energy deposit enough to paralyze them. As demonstrated in Enoto et al. [20], these signs may be interpreted as arrivals of a huge number of photons in a very short time interval of $\sim 1$ ms or less, implying the occurrence of a downward TGF. Figure 5 shows count histories of the short burst obtained by detector-B after the downward TGF. This part exponentially decays its intensity, with the decay constant of $\sim 60 \mathrm{~ms}$, which is consistent with a moderation time of fast neutrons produced via photonuclear reactions. [20]. Thus, this decay feature is also thought to be attributable to prompt gamma rays derived from neutron capture reactions and indicates photonuclear reactions take place in this event as well.

As described above, this observation shows a relation between lightning and the two types of high-energy emissions. Although we do not know if a gamma-ray glow always proceeds lightning and the subsequent gamma-ray emission originating from photonuclear reactions, such a possibility would be investigated by simultaneous observations of gamma rays and other wave-length such as radio ones. 


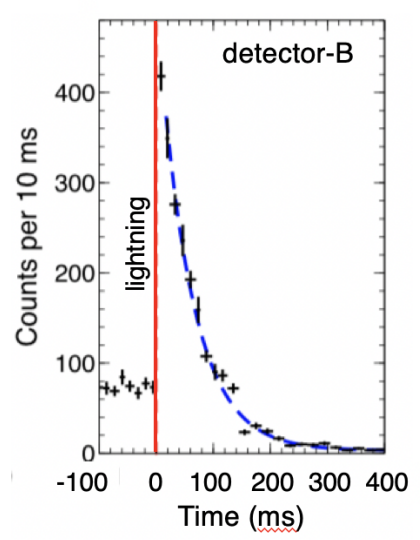

Figure 5: Count history in energy range of $0.4-20 \mathrm{MeV}$, obtained by detector-B in a period between -100 and $400 \mathrm{~ms}$ from lightning. A blue line shows an exponential function fitted to the experimental data. The function has a time constant of $59 \pm 2 \mathrm{~ms}$.

\section{Summary}

Since 2006, the GROWTH collaboration has performed high-energy radiation measurements during winter thunderstorms at the coastal area of Japan sea. As a result, we found two sorts of radiation bursts related to thunderstorms; one is a short burst lasting for 1s or less, and the other is a long burst typically lasting for $\sim$ a minute (or gamma-ray glow). Through those observations, we experimentally demonstrated that photonuclear reactions occurred in lightning. Furthermore, it was found that some short burst consists of initial bremsstrahlung emissions and the subsequent gammaray emissions originating from neutrons produced via photonuclear reactions. Just prior to these short bursts, gamma-ray glows were sometime detected. This implies that such a gamma-ray glow, or an underlying electron acceleration in a strong electric field cause lightning. Therefore, these high-energy gamma-ray observations would be a tool to better understand the lightning initiation that has been still argued as an unsolved problem.

\section{Acknowlegments}

This research is supported in part by JSPS/MEXT KAKENHI grants 15K05115, 15H03653, 16H04055, 16H06006, 16K05555, 17K05659, 18J13355, 18H01236, 19H00683, by Hakubi project and SPIRITS 2017 of Kyoto University, and by the joint research program of the Institute for Cosmic Ray Research (ICRR), the University of Tokyo.

\section{References}

[1] C.T.R. Wilson. Math. Proc. of the Cambridge Philos. Soc., 22(4):534-538, 1925.

[2] J. R. Dwyer, D. M. Smith, and S. A. Cummer. Space Sci. Rev., 173(1-4):133-196, 2012.

[3] A.V. Gurevich, G.M. Milikh, and R. Roussel-Dupre. Phys. Lett. A, 165(5-6):463-468, 1992.

[4] J. R. Dwyer. Geophy. Res. Lett., 30(20):8 1-8, 2003. 
[5] G. J. Fishman et al. Science, 264(5163):1313-1316, 1994.

[6] D. M. Smith et al. Science, 307(5712):1085-1088, 2005.

[7] M. McCarthy and G.K. Parks. Geophys. Res. Lett., 12(6):393-396, 1985.

[8] N. A. Kelley et al. Nature Comm., 6(1), 2015.

[9] V.V. Alexeenko, N.S. Khaerdinov, A.S. Lidvansky, and V.B. Petkov. Phys. Lett. A, 301(3-4):299-306, 2002.

[10] T. Torii, T. Sugita, S. Tanabe, Y. Kimura, M. Kamogawa, K. Yajima, and H. Yasuda. Geophys. Res. Lett., 36(13):L13804 1-4, 2009.

[11] H. Tsuchiya et al. Phys. Rev. Lett., 102(25):255003 1-4, 2009.

[12] H. Tsuchiya et al. Phys. Rev. D, 85(9):092006 1-14, 2012.

[13] M Amenomori et al. Proc. of 34rd Int. Cosmic Ray. Conf., (246):1-4, 2015.

[14] T. Torii, M. Takeishi, and T. Hosono. J. Geophy. Res.: Atmospheres, 107(D17):ACL 2-1-ACL 2-13, 2002.

[15] H. Tsuchiya et al. Phys. Rev. Lett., 99(16):165002 1-4, 2007.

[16] H. Tsuchiya et al. Journal of Geophysical Research, 116(D9):D09113 1-14, 2011.

[17] H. Tsuchiya et al. Phys. Rev. Lett., 111(1):015001 1-5, 2013.

[18] A. Chilingarian, G. Hovsepyan, G. Khanikyanc, A. Reymers, and S. Soghomonyan. EPL (Europhysics Letters), 110(4):49001, 2015.

[19] Y. Wada et al. Geophys. Res. Lett., 45:5700-5707, 2018.

[20] T. Enoto et al. Nature, 551(7681):481-484, 2017.

[21] Y. Wada et al. Commun. Phys., 2(67):1-9, 2019.

[22] L. M. Libby and H. R. Lukens. J. Geophys. Res., 78(9):5902-5903, 1973.

[23] G.N. Shah, H. Razdan, C.L. Bhat, and M. Ali. Nature, 313(1):773-775, 1985.

[24] L. P. Babich and R. A. Roussel-Dupre. Journal of Geophy. Res.: Atmospheres, 112(D13):D13303 $1-7,2007$. 